\title{
Measurement and meaningfulness
}

\section{J. van Brakel}

State University of Utrecht, Physics Laboratory. Foundations of Physics, 3508 TA Utrecht, The Netherlands

This is a reissue of a paper which appeared in ACTA IMEKO 1985, Proceedings of the $10^{\text {th }}$ World Congress of the International Measurement Confederation, "New measurement technology to serve mankind", 22-26.4.1985, Prague, pp. 319-333.

The question raised in this paper is: What is the meaning of a statement in which reference is made to measurements? Measuring a property of a system produces numbers. The question can be made more specific by considering the following aspects of these numbers: validity and purpose of numbers to be obtained; accuracy and stability of numbers obtained; possibility of obtaining numbers; meaningfulness of relations between numbers obtained; relation to numbers representing other properties; what is arbitrary and what not in what the numbers denote; in short: what do the numbers obtained in measurement signify.

\section{Section: RESEARCH PAPER}

Keywords: measurement theory, uncertainty

Citation: J. van Brakel, Measurement and meaningfulness, Acta IMEKO, vol. 3, no. 1, article 8, May 2014, identifier: IMEKO-ACTA-03 (2014)-01-08

Editor: Luca Mari, Università Carlo Cattaneo

Received May $1^{\text {st }}, 2014 ;$ In final form May $1^{\text {st }}, 2014$; Published May 2014

Copyright: @ 2014 IMEKO. This is an open-access article distributed under the terms of the Creative Commons Attribution 3.0 License, which permits unrestricted use, distribution, and reproduction in any medium, provided the original author and source are credited

\section{INTRODUCTION}

Consider statements in which reference is made to measured (quantified) properties of empirical systems, for example:

- the width of this piece of paper is approximately four prekik

- the viscosity of this fluidised bed is $600 \mathrm{cp}$

- the average pore size of this brick is $10 \mu \mathrm{m}$

- the poverty in the south of this country has increased drastically

Assuming that we know what is referred to by 'this' in (1)(4), can we specify criteria on the basis of which it can be decided whether such a statement is meaningful and what precisely its meaning is? The question of meaningfulness has been discussed in some detail in literature on the foundations of measurement (see references in [1], [2] and [3]). This discussion is related to and intertwined with discussions on the notions of scale invariance and dimensional invariance. In this paper no contribution to this mathematical theory of meaningfulness will be made. Instead the problem area "measurement and meaningfulness" will be placed in a wider perspective by investigating what conceptual problems arise if we simply raise the question what the meaning is of a statement involving measurements.

\section{DOES THE MEASUREMENT REPRESENT THE CONCEPT?}

A measurement always has a purpose; this purpose is part of the context in which the measurement is carried out [4]. Relative to this purpose the measurement has a validity and an accuracy (the terms 'validity' and 'accuracy' are used here in the sense that is common in the literature on psychometrics, e.g., the "validity of a test"). A measurement result is valid to the extent that it measures what it is meant to measure; it is accurate (and stable) to the extent that it measures what it measures accurately.

If the sole purpose of a measurement is to predict something (for example, a test to predict the performance of students at a particular examination), its validity is easy to assess (the test is a good one, is valid, if it predicts well; whether it predicts well is easy to assess). As long as we can make good predictions, we may not bother about what we are measuring. However, usually we do bother. Either, because we are concerned about the predictive validity of the measurement in other contexts. Or, because we explicitly require the measurement to measure something. This something is a concept (width, viscosity, pore size, poverty). In most cases we will say that the concept denotes properties of things in the real world. The examples (1)-(4) are chosen to draw attention to a number of different ways in which we may have to analyse the validity of a measurement statement: 
(a) The validity of (4) will depend on the normative, including political, motivations that are operative in specifying what we want the concept poverty to mean. For example: If we decide (should we?) to explain poverty in terms of income, do we mean income in money only or also other sorts of "income" (goods, services from other people)? Further, what do we require of the procedure by which the income data are processed into a measure of poverty? For example, it seems sensible to require that a transfer of income from a rich to a poor person (all other things remaining equal) should always reduce the measure of poverty - but until recently few poverty measures in actual use met that criterion [5].

(b) On first view it might seem that it is crystal clear what the concept of length is. However, what do we mean if we talk about the size of pores in a brick and we express this size on a length-scale. Numerous instruments and techniques are available which produce numbers which are said to represent the pore size (or the pore size distribution). But if we would investigate what information precisely is extracted from the brick by these instruments, it turns out that they all measure something different. If we would make photographs of cross sections through the brick, it is perfectly clear what we mean by referring to the distance between point $A$ and point $B$ (the length $A B$ ). It is also more or less clear what the different "porosimetric" instruments measure, but it is completely unclear what we mean by "pore size". Hence, the question of the validity of the instruments can only be assessed relative to whatever the measurement results are to be used for.

(c) The viscosity of a fluid bed is measured with the same type of instrument that is used to measure the viscosity of viscous liquids; data obtained are very reproducible. So there seems to be no problem here. But a fluidised bed is a mass of solid particles (for example sand) supported by a flowing fluid such that the particles do not form a fixed bed, but are in constant movement relative to one another. If we measure the so called viscosity of such s fluid-particles system, is this really the same sort of concept we are using when referring to the viscosity of liquids?

I hope the above examples give some idea of the way the meaning of a measurement statement depends on the context in which it is produced. The meaning of a measurement statement is related to the meaning of the concept it intends to represent and all uncertainties and vagueness inherent in a concept are not eliminated by having a procedure that produces "accurate" numbers in a reproducible way.

I won't discuss the relation of the concepts of accuracy and reproducibility to the meaning of measurement statements in any detail. For the moment, we can more or less circumvent these aspects by considering only the meaning of statements concerning one measurement, which is expressed on a scale chosen in such a way that there is no reasonable doubt about the exactness of the measurement. The following explanation of the meaning of (1) should illustrate this. As a unit of length I introduce the prekik. The distance between the two vertical bars in

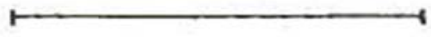

is equal to one prekik. Length measurements in prekiks are possible using the following instruction:
In order to measure the distance between two points, these two points have to be connected by a straight line and then it has to be counted how many times the standard of one prekik fits on that line

Many foundational problems are hidden in (6). For the moment (6) should be complemented with the following elucidations:

(i) It may be practical to make a number of copies of the standard or, using the standard, to construct a measuring rod on which there are little marks, the distance between subsequent marks being always one prekik. In this case we will say that we use different instruments, but all these instruments correspond to one measurement procedure, which is formulated in (6).

(ii) We do not consider parts of one prekik. (They don't exist so to say.) Then a distance in prekiks, for example (1), is exact if the distance is very large compared with the inaccuracies involved in the operational procedures referred to in (6) and (i) above. (There are some problems suppressed here, but we won't bother.)

(iii) We assume that the length of the standard does not change during transport. Whether this assumption is a convention of length measurements or whether this is a property of the empirical world is a subject of discussion in the foundations of relativity theory (see [6] and references given there).

\section{WHEN IS A MEASUREMENT POSSIBLE?}

In the previous section the basic assumption was made that we measure something. Before we start measuring we should have an idea what concept we intend to quantify through measuring. Such a quantification of a concept through measuring is only possible if the concept has certain properties. Let us assume that measurement is the representation of empirical structures on numerical structures (for the representational theory of measurement see for example [7] or [8]). Because the objects in the empirical structure are ordered in certain ways with respect to one of their properties, it is possible to represent this property in the numerical structure by assigning numbers to the objects in the empirical structure: the order in the empirical structure is mapped into the numerical structure. What does this mean? Consider the concept "mass" as an example:

(a) There is a concept "mass", the meaning of which is partly explained by telling what we mean if we say

$$
\mathrm{a}<_{\mathrm{m}} \mathrm{b}
$$

read: object $\mathrm{b}$ is heavier than object a. An operational definition of $<_{\mathrm{m}}$ refers for example to the use of a balance and tells us how to go about assessing whether in a particular case (7) is true or not. The explanation of what we mean by $<_{\mathrm{m}}$ is part of the meaning of any measurement statement about the mass of an object. If we know what we mean by $<_{\mathrm{m}}$ it is easy to define

$>_{\mathrm{m}}, \sim_{\mathrm{m}}, \geq_{\mathrm{m}}$, and $\leq_{\mathrm{m}}$. Note that it is irrelevant what instrument we use (asking somebody whether (7) is true or not in any particular case also counts as an instrument), except that we would like $<_{\mathrm{m}}$ to have certain properties. For example, it is advantageous if we can establish empirically that $\geq_{m}$ is 
connected and transitive. If this is so $\geq_{m}$ generates a weak order in the set of objects, $A_{m}$, which have the property mass.

(b) The meaning of the concept of mass is further explained by referring to the fact that an operation of physical concatenation ( $=$ adding together), ${ }_{\mathrm{m}}$, can be applied to elements of $A_{m}$. Just as in the case of $\geq_{m}$ it is attractive if ${ }_{m}$ has certain properties. For example it is advantageous if it has the properties of being associative and monotoneous. Whether this is so, is again a matter of empirical investigation.

(c) If $\geq_{\mathrm{m}}$ and $\circ_{\mathrm{m}}$ exist and have the properties mentioned, then it is usually possible to construct a standard sequence. In particular this is possible if the following holds

for any $e$ and $a$ in $A_{m}$ and any integer $k$, there exists an integer $\mathrm{n}$ such that

$$
\text { ne } \geq_{\mathrm{m}} \mathrm{ka}
$$

in which the meaning of ne is explained by

$$
1 \mathrm{e}=\mathrm{e} \text { and }(\mathrm{n}+1) \mathrm{e} \sim_{\mathrm{m}}\left(\mathrm{ne} \circ_{\mathrm{m}} \mathrm{e}\right)
$$

and similarly for ka. It is not obvious in what way we should consider (8) a condition which has to be fulfilled empirically. Also there are writers who object to using such "Archimedean axioms" in the first place (see [9] and [10], a review of [7]). However, I pass that over. If something like (8) holds and

$\geq_{\mathrm{m}}$ and $\circ_{\mathrm{m}}$ have the properties as mentioned, we can construct a standard sequence for mass, which allows us to assess the relative mass of objects as follows. Let us choose for e the standard kilogram in Paris. First we make many copies of e, so that we are never at a loss to construct an ne. Assume further that we want to determine the mass of President Reagan. For simplicity we choose $\mathrm{k}=1$ and then we find the smallest value of a for which

$$
\text { ne }>_{m} a_{m} \geq_{m}(n-1) e
$$

where $a_{m}$ represents Reagan with respect to his mass. Equation (10) fixes the mass of Reagan relative to that of the standard kilogram in Paris. Ideally, we would like to be able to find

$$
\text { ne } \sim_{\mathrm{m}} \mathrm{a}_{\mathrm{m}}
$$

This could be so by accident, but usually it is not the case. However, we can approach an equivalence by choosing a large value of $\mathrm{k}$ (for which purpose we have to make $\mathrm{k}$ copies of Reagan, at least k copies of Reagan's mass). Of course, this is not the way we actually determine the mass of an object. Nevertheless, this is part of what it means if we say that a property like mass can be measured.

(d) Note that until now, we have done nothing more than counting the number of identical copies, manipulating objects and assessing whether $>_{\mathrm{m}}$ or $\sim_{\mathrm{m}}$ applies. Because all this is possible, we can measure mass on a scale $\Phi$, which is, in this case, an additive function from $A_{m}$ to the real numbers, and, by convention, $\Phi(\mathrm{e})=1$.

Many questions can be raised in connection with this account; I mention only two clusters of questions: (i) Operational definitions of $\geq_{m}$ and $\sim_{m}$ have been given referring to a balance. However, we cannot put the earth or an electron on a balance. How is the mass of these objects related to the standard kilogram in Paris? Similarly, on a strictly operationalistic point of view, the ten or so different methods to measure stellar distances, would all measure different properties or concepts. But this is not what we want, because most of us would believe that there is just one distance between two stars.

(ii) I said, if $>_{\mathrm{m}}$ and $\circ_{\mathrm{m}}$ have certain properties and (8) holds, then mass can be measured on an additive scale $\Phi$. But what do we do if we find an exception, for example, in a particular case we observe that $>_{\mathrm{m}}$ is not transitive or $\circ_{\mathrm{m}}$ is not associative. Why are we so sure this must be due to inaccuracies in our instruments or our observations? (in fact, in modern physics mass is not "additive" and [11] has proposed to drop mass as a basic unit and to replace it by the "quantum of action")

I have used mass as an example, because this is by far the simplest case: for length there are alternative interpretations for $\circ_{1}$ and we have to account for the three dimensionality of space (or four dimensionality of space-time); for time the definition of $o_{t}$ and the choice of a unit is more problematic; for force we have to take into account the direction; velocity has an upper and a lower bound (at least that is what we think, do we?), which presents special problems; and so on (for references to literature on some of these questions see [4]). But always, it is part of the meaning of a measurement statement, what the interpretation is of $>$ and $\circ$ for the particular concept (or why there is no $\circ$, if there is no such operation).

\section{MEANINGFULNESS OF STATEMENTS INVOLVING A COMPARISON OF MEASUREMENTS}

On an abstract level we can say: Meaningful numerical statements are those that remain invariant under permissible changes in the representation. What does this mean? Consider the following measurement statements:

the average radius of the earth is $6371 \mathrm{~km}$

the average radius of the earth is $3960 \mathrm{mi}$

A quantity such as mass or length can be measured on different scales. Because of the arbitrary choice of the standard e, any scale $\Phi$ is invariant with respect to transformations of the sort

$$
\Phi^{\prime}=\alpha \Phi
$$

where $\alpha$ is a real number. We encounter such an $\alpha$ if we transform (12) into (13) or if we transform prekiks into meters. In that sense the change of (12) to (13) (or vice versa) is meaningful. But there is another question: statement (12) is concerned with the average radius. The number 6371 is the result of manipulations with other numbers. What is the meaning of such an average? In the first place there is the question of validity discussed in section 2: Which distances have to be averaged? Answer (say): Connect every point on the surface of the earth with its centre. Questions: Where is the centre? What sort of concept does this average represent? Secondly, if we have agreed on the measurement data that have to be averaged, are we allowed to do that? This question can be given an exact answer in the representational theory of 
measurement as follows (for this technical concept of meaningfulness see [1], [12], [13], and references given there; the concept was introduced in a systematic way for the first time by [14]). A statement in which something is said about the relation between two averages (or any other manipulation of measurement data) is meaningful, if the truth-value of this statement does not change if we choose another scale to express the original data. For example, with $\Phi$ a scale for length, consider:

$$
\frac{1}{\mathrm{n}} \sum_{\mathrm{i}=1}^{\mathrm{n}} \Phi\left(\mathrm{a}_{\mathrm{i}}\right)>\frac{1}{\mathrm{~m}} \sum_{\mathrm{i}=1}^{\mathrm{m}} \Phi\left(\mathrm{b}_{\mathrm{i}}\right)
$$

If we substitute first $\Phi^{\prime}$ and then (14) in (15) we obtain:

$$
\frac{1}{\mathrm{n}} \sum_{\mathrm{i}=1}^{\mathrm{n}} \alpha \Phi\left(\mathrm{a}_{\mathrm{i}}\right)>\frac{1}{\mathrm{~m}} \sum_{\mathrm{i}=1}^{\mathrm{m}} \alpha \Phi\left(\mathrm{b}_{\mathrm{i}}\right)
$$

This is equivalent to (15); hence (15) is meaningful. Another example of a meaningful statement for length is:

$$
\Phi(\mathrm{a})=\mathrm{n} \Phi(\mathrm{b})
$$

with $\mathrm{n}$ an integer. Although this may seem trivial, please note that for temperature measured on an interval scale (such as ${ }^{\circ} \mathrm{C}$ or ${ }^{\circ} \mathrm{F}$ ), (16) is meaningful, but not (17). For wind force measured on the scale of Beaufort or hardness measured on Mohs scale, neither (16) nor (17) is meaningful.

All physical quantities which are embedded in general theories are measured on ratio scales and usually we are completely familiar with what are meaningful statements and what not. We know we can compare averages and we see at once that

$$
\Phi(\mathrm{a})+\Phi(\mathrm{b})=[\Phi(\mathrm{c})]^{2}
$$

is not meaningful. However, in the social sciences, in psychology, and in the technical sciences there are many isolated quantities, which often are not measured on ratio scales or where there are other hidden traps. Consider for example a price index defined as:

$$
\mathrm{P}=\sum \mathrm{q}_{\mathrm{i}}^{0} \mathrm{P}_{\mathrm{i}}^{0} / \sum \mathrm{q}_{\mathrm{i}}^{0} \mathrm{P}_{\mathrm{i}}^{1}
$$

Here $\mathrm{q}_{\mathrm{i}}^{0}$ is a chosen consumption pattern (say $10 \mathrm{~kg}$ potatoes, 2 eggs, 0.01 television set, and so on) and $\mathrm{P}_{\mathrm{i}}^{0}$ and $\mathrm{P}_{\mathrm{i}}^{1}$ are the prices at times $t_{0}$ and $t_{1}$ respectively. In its generality $P$ is not meaningful, because whether $\mathrm{P}$ decreases or increases depends on the choice of $q_{i}^{0}$ (more detailed examples in [15]).

\section{DERIVED MEASUREMENT AND DIMENSIONAL INVARIANCE}

Why do we see at once that (18) is not meaningful? This is because (18) is not dimensionally homogeneous. Various explanations or theories have been proposed to explain why numerical laws should be dimensionally homogeneous (see [7], pp. 504-512, [16] and [17]). The explanation that has been worked out in most detail is the explanation according to which the requirement of dimensional invariance follows directly from the concept of meaningfulness defined in the previous section and the properties of the qualitative representations of the numerical laws ([2]; see also references in [1] and [3]). (Here "qualitative" means: representing the laws without using scales.) There is no room to discuss the subject of dimensional invariance in detail here. However, one aspect I would like to draw attention to. It has been argued in the literature on this subject that because of these criteria of invariance or meaningfulness, the possible forms of a law are extremely limited (for the first time by [3]; most recently by [18]; invariance requirements have also been invoked to solve paradoxes in the a priori estimation of equal probabilities, like the Bertrand paradox: see [19] and references given there). For example, we do not only know that

$$
\mathrm{E}=\mathrm{m}^{2} \mathrm{c}
$$

is not a law of nature (because it is $\mathrm{E}=\mathrm{mc}^{2}$ ). We know more, it is argued. We know for certain that (20) cannot be a law of nature. This fact has been of use for a long time in the practice of dimensional analysis. However, this "setting limitations on the possible forms of the law" should not be exaggerated, because the criterion only holds if we are certain that we know all the variables that enter into the law. But if we still have to discover the law, how can we be so certain that we know all the variables that enter into the law? Moreover, if one of the variables is dimensionless, or two or more (but not all) of the variables can be combined into a dimensionless group, then the dimensionless part can occur in the law as the argument of any mathematical function.

In the applied sciences, laws often have "empirical" forms. An example is

$$
\frac{\mathrm{dU}}{\mathrm{dL}}=-C L^{\mathrm{n}}
$$

in which $\mathrm{C}$ and $\mathrm{n}$ are constants, and $\mathrm{dU}$ is the energy required to effect a small change $\mathrm{dL}$ in the size of a unit of material in a size reduction process (as in a mill). The value of $\mathrm{n}$ is different for different materials. In practice values of 1.5 or 2 are often used for $\mathrm{n}$. It is obvious that in this case the requirement of dimensional invariance sets very few limitations on the form of the law, because $\mathrm{C}$ is only defined by its occurrence in this law and absorbs whatever dimension is generated by the value of $n$. The fact that the experimentally determined value of $\mathrm{n}$ is not a whole number is no problem, because experimentally we can at best determine the value of rational numbers (as distinct from real numbers) and if $\mathrm{n}$ is a fraction, we can always eliminate the denominator. Moreover, because $\mathrm{C}$ is not further interpreted,we can make all terms dimensionless by dividing $\mathrm{U}$ and $\mathrm{L}$ by a reference value which has the same dimensions. In a way this is what happens in kinetic laws such as the law of radioactive decay:

$$
\mathrm{N} / \mathrm{N}_{0}=\exp (-\lambda \mathrm{t})
$$

The decay constant is the inverse of a reference value for $t$. Equation (22) makes sense, because we have a particular interpretation for $\lambda$. But if we would not have that interpretation, any functional relationship between $\mathrm{N} / \mathrm{N}_{0}$ and $\lambda \mathrm{t}$ would be allowed. 
Another example of a "pseudo-law" is:

$$
\mathrm{S}=\alpha \mathrm{t}^{\beta}
$$

where $\mathrm{S}$ is the tension (at constant strain), $\mathrm{t}$ is time and $\alpha$ and $\beta$ are constants for a particular material. The so called anomalous character of (23) has generated a whole discussion in the literature (this discussion has been generated by a peculiar presentation to the Royal Society in London [20]; see also [17], pp. 45-46, and [7], p.464), in which doubts have been raised concerning the Newtonian definition for equality of time intervals and the parameter $\alpha$ has been ascribed the dimensions "antilog $(\log \mathrm{L} \log \mathrm{T})$ ". However just as in (22) the constant of proportionality has no meaning independent of the equation in which it occurs. Hence, we might as well write instead of (23):

$$
\mathrm{S}=\mathrm{pe}^{-\mathrm{t} / \tau}+\mathrm{r}
$$

which solves all problems of awkward dimensions (If the fit of (24) is not as good as that of (23) we add one more exponential term.)

It may be of interest to note that (23) is of some historical interest, if it is read as an equation describing the falling distance of an object as a function of time (in the seventeenth century various laws were proposed to describe "Galilei's law". In that context Chr. Huygens was probably the first to argue that certain laws could be excluded a priori).

\section{STANDARDS OF MEASUREMENT}

Consider the following statements:

$$
\begin{aligned}
& 1 \mathrm{~m}=1000 \mathrm{~mm} \\
& 1 \mathrm{~m}=19.2 \text { prekik } \\
& 1 \mathrm{~m}=\text { the length of the standard meter in Paris }
\end{aligned}
$$

If we would analyse the meaning of each of (25) - (27) we would see that they are three quite different kinds of statements. I won't go into the "philosophical" details of this here, except for drawing attention to one particular peculiarity of standards of measurement in the contribution they make to the meaning of a measurement statement. I have said that the meaning of an object having a mass of $10 \mathrm{~kg}$ can be partly explained by saying that the mass of this object is equivalent $\left(\sim_{\mathrm{m}}\right.$, not $\left.=\right)$ to the physical concatenation of 10 standard kilograms each of which is equivalent in mass to the standard kilogram kept in Sèvres near Paris. The meaning of the measurement statement is so to say explained by pointing to a certain relation the object of the measurement statement bears to the standard. Now assume that the building in Sèvres, including the standard kilogram which is kept there, is completely destroyed in a fire. Does this mean that, when this happens, instantaneously all statements giving measurement results in $\mathrm{kg}$ become meaningless? Presumably not, but what then is the meaning of these statements?

\section{CONCLUDING REMARKS}

In the previous sections I have only touched upon some aspects that have to be considered when we pose the question what the meaning is of a measurement statement. I have emphasised what I think are important aspects and I have used up a lot of space giving examples from the practice of measurement. In a further systematic development of the points raised here, it will be necessary, I think, to do this within the terminological framework of philosophical theories of meaning. This would include (i) analysis of the meaning of measurement statements using the Fregean concepts of sense and reference (which in the context of measurement might be called "operational" and "factual" meaning), (ii) taking into account recent philosophical discussions concerning the question whether there are statements which are a priori and contingent or statements which are a posteriori and necessary (as this may shed light on the distinction between what is fact and what is convention in measurement) and (iii) an analysis of the different sorts of identity or equivalence that are presupposed in measurement statements. (These three aspects are all interrelated in a theory of meaning). On the applied side, a further development of the points raised in this paper would also require analysis of a number of "cases", to make clear which quantities are measured on what scales. For example, most scientists would probably say that density is measured on a ratio scale, this is not self-evident and we may well have to conclude that density is measured on an interval scale [16]. And to give a last example, in the design of grinding mills for coal the Hardgrove Grindability Index is used to predict the energy consumption of the mill for a given specification of the size reduction to be achieved. This index can be interpreted to be some sort of measure of the material constant $C$ in equation (21). But if we analyse a large set of data for this index, it is dubious whether we can even say that the grindability is measured on an ordinal scale: Different samples from the same coal-seam have different grindabilities. There is no correlation between the grindability of a coal and its rank, or its composition. Also, there is no correlation between the grindability as determined in the standard mill used to determine the hardgrove index and the grindability as determined in another standard mill (for example Bond's index, in which case $\mathrm{n}$ in eq. (21) is 1.5 instead of 2). Furthermore there is no relation between the grindability and the modulus of elasticity of coal or the hardness of coal determined by some standard test (for example the Brinell test). So what do we mean if we say that the grindability has been determined to be, say, 60?

No doubt some of the considerations involved in a study concerning the meaning of measurement statements as suggested above, will be rather abstract or philosophical, but I belief such a study could make an important contribution to a better understanding of what measurement is by giving a conceptual analysis of the meaning of measurement statements.

\section{REFERENCES}

[1] D.H. Krantz et al, Foundations of measurement, vol. 2 (to be published, typescript consulted dated May 1979).

[2] R.D. Luce, Dimensionally invariant numerical laws correspond to meaningful qualitative relations, Phil. Sci., 45 (1978) pp.1-16.

[3] R.D. Luce, On the possible psychophysical laws, Psychol. Rev., 66 (1959) pp.81-95.

[4] J. van Brakel, Norms and facts in measurement, Measurement, 2 (1984), pp.45-51.

[5] A. Sen, Poverty: An ordinal approach to measurement, Econometrica, 44 (1976), pp. 219-231.

[6] W. Balzer, A. Kamlah, Geometry by ropes and rods, Erkenntnis, 15 (1980), pp. 245-267.

[7] D.H. Krantz et al, Foundations of measurement, vol.1, New York, 1971.

[8] F.S. Roberts, Measurement theory, Reading (Mass.), 1979. 
[9] J.O. Ramsay, Algebraic representation in the physical and behavioral sciences, Synthese, 33 (1976), pp. 419-453.

[10] J.O. Ramsay, Review of Foundations of Measurement, Psychometrika, 40 (1975), pp. 257-262.

[11] E.J. Post, Physical dimensions and covariance, Found. Physics, 12 (1982), pp. 169-195.

[12] L. Narens, Theory and decision, 13 (1981), pp.1-70.

[13] F.S. Roberts, On Luce's theory of meaningfulness, Phil. Sci, 47 (1980), pp.424-433.

[14] J. Pfanzagl, Theory of measurement, Würzburg, 1968.

[15] D.K. Osborne, Unified theory of derived measurement, Synthese, 33 (1976), pp.455-481.
[16] D.K. Osborne, On dimensional invariance, Quality and Quantity, 12 (1978), pp.75-89.

[17] J. Palacios, Dimensional Analysis, London, 1964.

[18] J.C. Falmagne, L. Narens, Synthese, Scales and meaningfulness of quantitative laws, 55 (1983), pp.287-325.

[19] P. Milne, A Note on Scale Invariance, Brit. Jour. Phil. Sci., 34 (1983), pp.49-55.

[20] G.W.S. Blair et al, Limitations of the newtonian time scale in relation to non-equilibrium rheological states and a theory of quasi-properties, Proc. of the Royal Society of London, Series A-Mathematical and Physical Sciences, 189 (1947), pp.69-87. 